\title{
Sunflower seed mimicking complicated hydatid cyst
}

\author{
A. Fındıkcıoglu, D. Kilic, A. Hatipoglu
}

\author{
ABSTRACT: Sunflower seed mimicking complicated \\ hydatid cyst. A. Findıkcioglu, D. Kilic, A. Hatipoglu. \\ We present a case of a foreign body in lung parenchy- \\ ma, found incidentally during surgery in an adult woman. \\ The clinical and radiological findings were consistent with
}

\author{
a pulmonary hydatid cyst. Owing to the similar clinical \\ and radiological appearances, a thoracotomy was re- \\ quired for the correct diagnosis of a sunflower seed mim- \\ icking hydatid disease. \\ Monaldi Arch Chest Dis 2006; 65: 2, 116-117.
}

Keywords: Foreign body, cyst hydatid, lung parenchyma.

Baskent University, Faculty of Medicine, Department of Thoracic Surgery, Adana, Turkey.

Correspondence: Alper Findikcioglu MD; Baskent University Faculty of Medicine; Department of Thoracic Surgery; Adana Teaching and Medical Research Center; Dadaloglu Mah. 39. Sokak No:6, Yuregir; 01250 Adana, Turkey; e-mail: alperfindikci@hotmail.com

Aspirated foreign bodies in adults may remain hidden until accidentally found during exploratory surgery. Symptoms that should raise suspicion of an inhaled foreign body include coughing, dyspnea, choking, wheezing, chest pain, cyanosis, hemoptysis, and fever. In adults, however, these symptoms alone are usually insufficient to suspect a foreign body unless there is some history of aspirating a foreign body. Usually, a bronchoscopy leads to a diagnosis of aspirating a foreign body; however, this was not the case with the current patient. With our patient, the radiological and clinical findings were nonspecific. The patient's history revealed a previous operation for a hydatid cyst in the liver, which led to a diagnosis of complicated hydatid cyst. It was only during surgery, however, that a sunflower seed was found.

\section{Case Report}

A 30-year-old woman presented with complaints of chest pain on the right side and a non-productive cough of 2 months' in duration. Apart from a temperature of $37.5^{\circ} \mathrm{C}$, the results of her physical examination were normal. No evidence of psychiatric disorders was present. Her history was significant for an operation 5 years earlier owing to a hydatid cyst in the liver. Her family history revealed that her mother was operated on for hydatid cysts in the liver and lung. Laboratory tests revealed no abnormalities except for an erythrocyte sedimentation rate of $22 \mathrm{~mm} /$ hour. Additionally, an ELISA test was positive for hydatid disease. The sputum culture did not indicate any pulmonary infection.

A chest radiograph revealed a smooth, rounded mass on the inferior area of the right lung. Contrastenhanced computed tomographic imaging of the chest revealed a $5 \times 5 \mathrm{~cm}$ bi-lobed cystic lesion of homogenous density, with smooth boundaries, in the superior segment of the right lower lobe (figure 1). In addition to the cystic lesion, multiple nodular satellite lesions of the inferior lobe and limited bronchiectasis of the medial segment of the right middle lobe were evident (figure 2). In addition, 1 cystic lesion was observed in the seventh segment of the liver.

The fiberoptic bronchoscopy demonstrated diffuse inflammatory changes spreading to the mucosa of the intermediate and right lower-lobe bronchus. We did not obtain a diagnostic result with bronchoalveolar lavage culture and cytology.

A right posterolateral thoracotomy revealed an abscess with a thick, pus-filled lesion in the superior segment of the right lower lobe. The lesion was separated into two segments by a thin membrane. After opening and draining the abscess, a $2-\mathrm{cm}$ foreign body with a rough surface was found in the cavity (figure 4). Neither a bronchial air leak nor a relationship between the lesion and the tracheobronchial tree was observed. A medial segmentectomy was performed for the bronchiectasis in the middle lobe, and a wedge resection was performed for the nodular satellite lesions in the parenchyma. The other lesion, the hydatic cyst in the liver, was removed via a transdiaphragmatic approach. The patient was discharged on the seventh post-operative day with no complications. Her post-operative course was uneventful at six months' follow-up.

\section{Discussion}

Echinococcus infection in the lung parenchyma can present with various symptoms, signs, and radiological appearances. Typical radiological imaging of the hydatic cyst is a smooth, rounded, cystic lesion of a water-like consistency; however, complicated type of the cyst may resemble bronchial carcinoma or a lung abscess [1]. On the other hand, pulmonary lesions may appear as hydatid disease in endemic regions, owing to non-specific clinical and radiological findings of the cyst. 


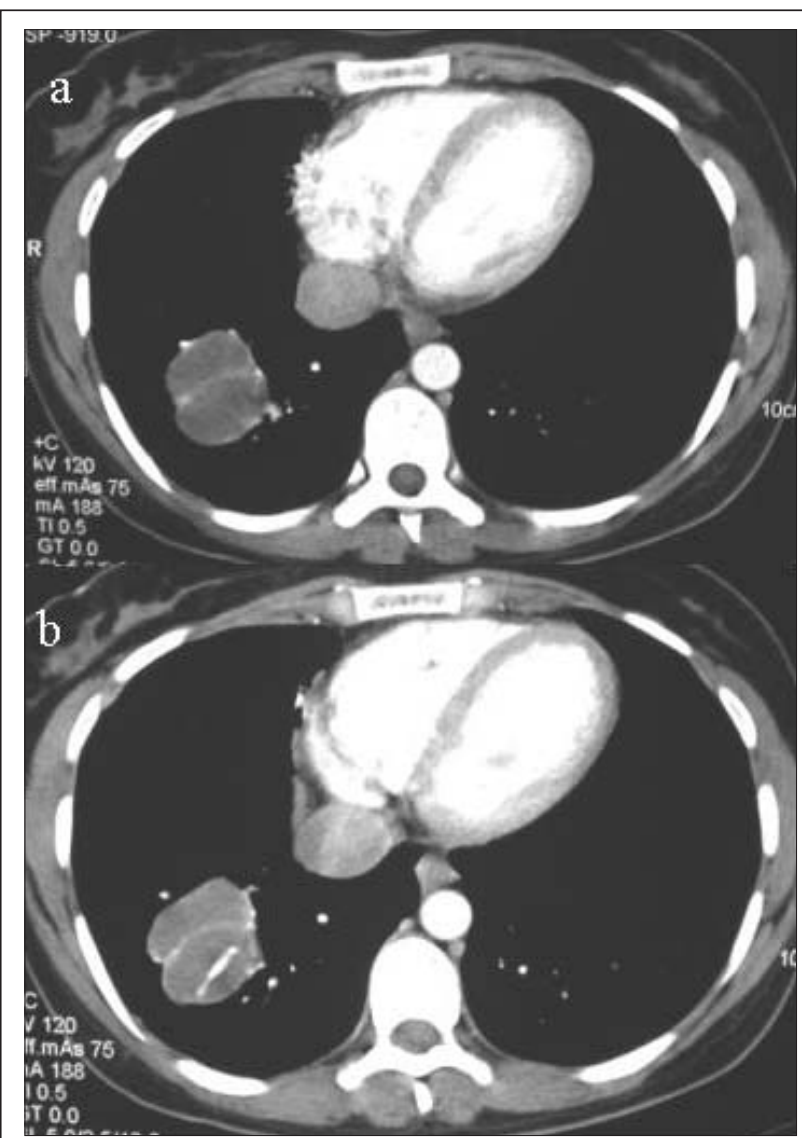

Fig. 1. - CT scan of the lesion in the right lower lobe consistent with a hydatid cyst (a). The scan shows a linear calcification in the middle of the cyst (b).

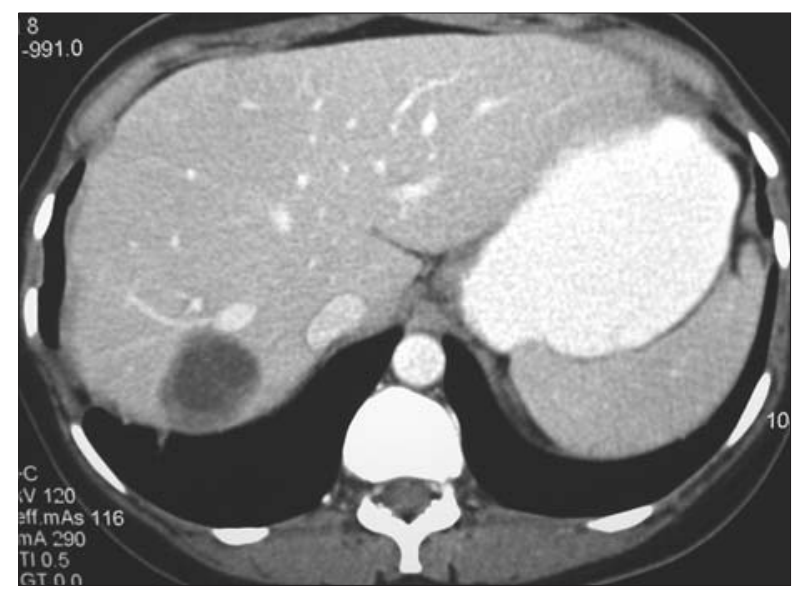

Fig. 3. - CT scan of the hydatid cyst in the liver.

A bronchoscopy may be used as the first choice for diagnosing and removing an aspirated foreign body [2]. However, most cases of hydatid cyst do not require bronchoscopy. In cases where a germinative membrane has been aspirated, bronchoscopic intervention may be necessary. In addition, a bronchoscopy may be used to differentiate between malignancies and hydatid disease. Any delay in diagnosing a tracheo-bronchial foreign body can lead to significant morbidity in children [3]. However, foreign body aspiration into lung parenchyma in adults, is rare. The chronic complications of prolonged foreign body impaction include bronchiectasis. Although rare, tracheo-bronchial aspiration of a foreign body in adults can occur in a variety of clinical settings. Strong clin-

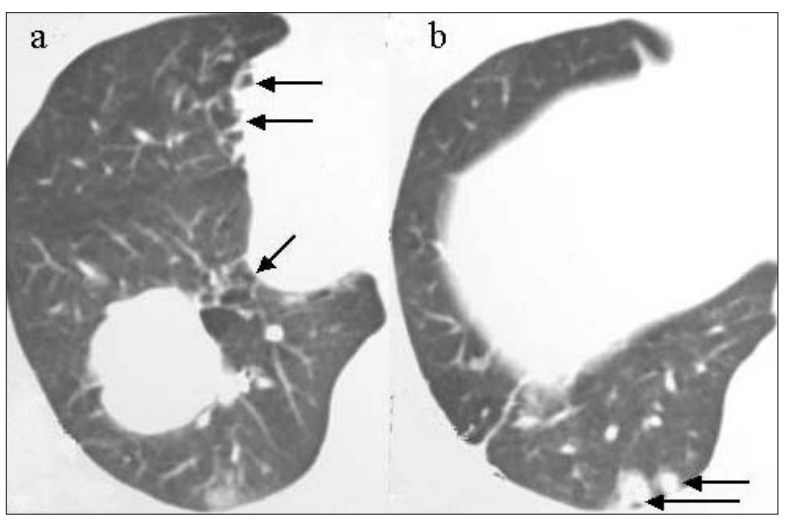

Fig. 2. - Bronchiectasis of the middle and lower lobe (a) and satellite lesions in the right lower lobe (b) (black arrows).

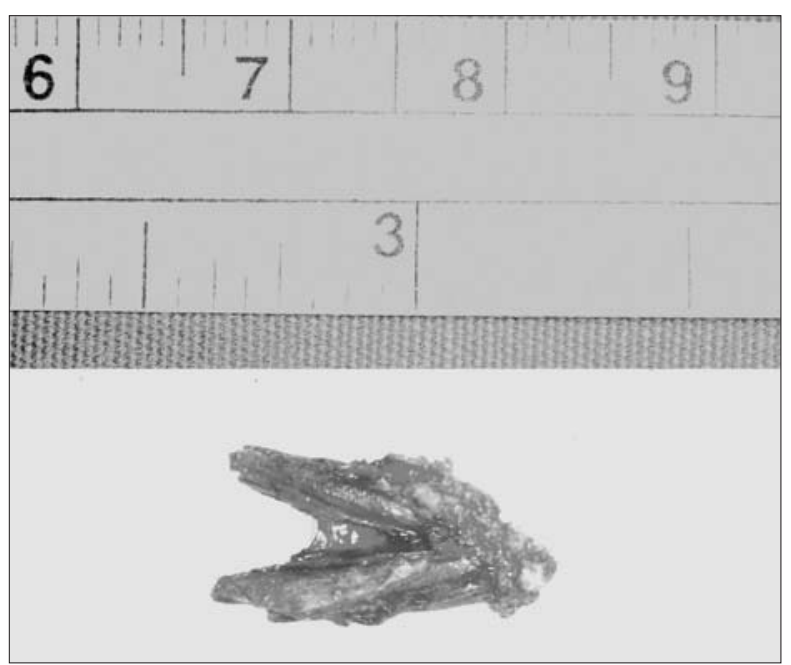

Fig. 4. - The foreign body (sunflower seed).

ical suspicion is necessary for diagnosis [4]. Early diagnosis and appropriate treatment for inhaled foreign bodies are important to prevent complications that could endanger the patient's life [5]. Physicians must be aware of the possibility of a foreign body in lung parenchyma in the patient with bronchiectasis.

The finding of a roasted sunflower seed instead of a cyst membrane was surprising. In this case, a bronchoscopy could not lead to the diagnosis and treatment of an aspirated foreign body with non-specific lesions. However, surgery remains an unequivocally diagnostic and therapeutic procedure for pulmonary lesions when a bronchoscopy is not specific.

\section{References}

1. Kilic D, Tercan F, Şahin E, Bilen A, et al. Unusual radiologic manifestations of the echinococcus infection in the thorax. J Thorac Imaging 2006; 21: 32-3.

2. Liebler JM, Markin CJ. Fiberoptic bronchoscopy for diagnosis and treatment. Crit Care Clin 2000; 16: 83-100.

3. Saquib Mallick M, Rauf Khan A, Al-Bassam A. Late presentation of tracheobronchial foreign body aspiration in children. J Trop Pediatr 2005; 51: 145-148.

4. Limper AH, Prakash UB. Tracheobronchial foreign bodies in adults. Ann Intern Med 1990; 112: 604-609.

5. Yurdakul AS, Hoca NT, Gokcek A, Cimen F, Cakaloglu A, Atikcan S. An interesting cause of recurrent haemoptysis: haemoptysis 7 years after a foreign body penetrated the lung parenchyma and aorta. Respirology 2005; 10: 229-232. 